\title{
CORRELAÇÃO ENTRE O COEFICIENTE DE EXPANSÃO TÉRMICA E O TEOR DE SÓLIDOS TOTAIS PARA O SUCO DE ABACAXI
}

\section{CORRELATION BETWEEN THE THERMAL EXPANSION COEFFICIENT AND TOTAL SOLIDS CONTENT FOR PINEAPPLE JUICE}

\author{
César Augusto Canciam ${ }^{1}$ \\ ${ }^{1}$ Universidade Tecnológica Federal do Paraná - UTFPR - Ponta Grossa - Brasil canciam@ utfpr.edu.br
}

\begin{abstract}
Resumo
O conhecimento do coeficiente de expansão térmica de uma substância é importante para avaliar o impacto da dilatação térmica sobre a medição volumétrica. Para produtos de frutas tropicais, como o suco de abacaxi, não existe referências na literatura sobre o coeficiente de expansão térmica. Neste sentido, o presente trabalho teve por objetivo verificar se existe uma correlação linear entre o coeficiente de expansão térmica do suco de abacaxi e o teor de sólidos totais. Para tanto, o coeficiente de expansão térmica foi obtido a partir da modelagem matemática de dados da densidade do suco de abacaxi em função da temperatura e do teor de sólidos totais. O coeficiente de expansão térmica

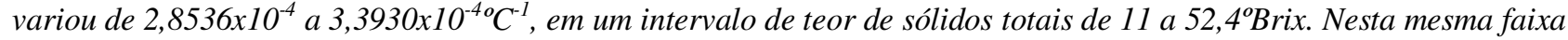
de teor de sólidos totais, verificou-se que existe uma correlação linear entre o coeficiente de expansão térmica e o teor de sólidos totais, com um coeficiente de correlação próximo da unidade, classificando a correlação linear como muito forte.
\end{abstract}

Palavras-chave: dilatação, sólidos totais, correlação.

\section{Introdução}

A indústria de alimentos tem grande interesse pelas frutas tropicais, pois estas apresentam sabores e aromas característicos. Por estas características aliadas ao desenvolvimento tecnológico de conservação dos atributos sensoriais de seus produtos, o consumo de sucos de frutas tropicais vem aumentando significativamente em todo mundo (MARCELLINI et al., 2006).

Dentre as frutas tropicais, o abacaxi (Ananas comusus L. Merril) apresenta destaque, principalmente pela qualidade sensorial diferenciada e pelo potencial de exportação em expansão (MARCELLINI et al., 2006). De acordo com Marin e colaboradores (2012), com base nos dados da Organização das Nações Unidas para Agricultura e Alimentação (FAO) em 2005, o Brasil é o maior produtor mundial de abacaxi. Filipinas, Tailândia, Índia e China ocupam, respectivamente, $2^{\circ}, 3^{\circ}, 4^{\circ}$ e $5^{\circ}$ lugares na produção mundial. Estes cinco países produtores respondem por mais de $53 \%$ da produção mundial desta fruta. Com exceção da Tailândia e Filipinas, as produções são destinadas 
basicamente ao mercado interno. O Brasil participa, timidamente, do comércio internacional de abacaxi.

As principais variedades de abacaxi com importância comercial são Smooth Cayenne, Singapure Spanish, Red Spanish e Selangor Green (MARCELLINI et al., 2006). No Brasil, destacam-se a variedade Pérola (com cerca de $80 \%$ da área total cultivada de abacaxi) e Smooth Cayenne (com cerca de $20 \%$ da área total cultivada). A variedade Smooth Cayenne, apesar de apresentar a menor área cultivada no país, é a variedade preferida por europeus e americanos (MARIN et al., 2012). No Brasil, são também cultivadas as variedades Boituva, Primavera, Perolera e cultivar IAC Gomo-de-mel (GRANADA et al., 2003).

O Ministério da Agricultura, Pecuária e Abastecimento (MAPA), desde 2002, regulamenta a comercialização de frutos de abacaxi para todo o território nacional. Esta legislação determina que o teor mínimo de sólidos totais seja de 12 Brix, além de critérios de tamanhos e graus de maturação aparente dos frutos (REINHARDT et al., 2004).

A composição química do abacaxi depende do estágio de maturação e de fatores agronômicos e ambientais. De um modo geral, a produção de abacaxi no Brasil ocorre no período do verão, o que acarreta em frutas com maior teor de açúcares e menor acidez (COUTO, 2008).

O MAPA define o suco de abacaxi como uma bebida não fermentada e não diluída, obtida da parte comestível do abacaxi. No suco de abacaxi, a legislação determina que o teor mínimo de sólidos totais seja de $11^{\circ}$ Brix (a $20^{\circ} \mathrm{C}$ ) (PINHEIRO et al., 2006). Na Europa, os sucos de abacaxi comercializados devem conter no mínimo 40\% de polpa de abacaxi (COUTO, 2008). Nos países europeus, o suco de abacaxi é normalmente preparado pela diluição do suco concentrado de abacaxi, importado de regiões tropicais e subtropicais (MARCELLINI et al., 2006).

O teor de sólidos totais (medidos em ${ }^{\circ}$ Brix) indica a quantidade de substâncias que se encontra dissolvida no suco, sendo constituída, em sua maioria, por açúcares (glicose, frutose e sacarose) (CHAVES et al., 2004).

Com relação a outros compostos dissolvidos, Couto (2008) comenta que o suco de abacaxi fresco é caracterizado por conter alto teor de polpa, ácidos orgânicos (em sua maioria, ácido cítrico e ácido málico), potássio, magnésio, cálcio, vitamina $\mathrm{C}$ e compostos voláteis responsáveis pelo aroma característico. No caso do abacaxi, a relação entre as concentrações de ácido cítrico e ácido málico é igual a 2 e a relação frutose/glicose igual a 1, ou seja, a quantidade de frutose somada com a de glicose dividida pela concentração de sacarose deve ser igual a 1. Estes parâmetros são aceitos somente para o suco de abacaxi.

Segundo Callister (2008), o coeficiente de expansão térmica ( $\beta$ ), a capacidade calorífica e a condutividade térmica são consideradas as propriedades térmicas dos materiais, pois estão relacionadas com a reação dos materiais devido à aplicação de calor. 
O coeficiente de expansão fornece uma medida da variação da densidade em resposta a uma mudança na temperatura, sob condição de pressão constante (INCROPERA e DEWITT, 2008).

A equação (1) expressa o coeficiente de expansão térmica em função da variação da densidade $(\rho)$ e da variação da temperatura $(T)$, mantendo a pressão $(P)$ constante (ZURITZ et al., 2005).

$$
\beta=\left(\frac{-1}{\rho}\right) \cdot\left(\frac{\partial \rho}{\partial T}\right)_{P}
$$

Integrando a equação (1), a equação (2) relaciona $\ln \left(\rho_{o} / \rho\right)$ com o coeficiente de expansão térmica e a diferença entre as temperaturas final $(T)$ e inicial $\left(T_{0}\right)$ (CANCIAM, 2005):

$$
\ln \left(\rho_{0} / \rho\right)=\beta \cdot\left(T-T_{0}\right)
$$

Na equação (2), $\rho_{0}$ e $\rho$ correspondem, respectivamente, à densidade na temperatura inicial e à densidade na temperatura final.

Assim, com base nos dados da densidade em função da temperatura, consegue-se determinar o coeficiente de expansão térmica (SANTOS e VIEIRA, 2010).

Segundo Canciam (2010), a equação (2) corresponde a uma função afim. Dessa forma, o gráfico de $\ln \left(\rho_{0} / \rho\right)$ versus $\left(T-T_{0}\right)$ fornece uma reta, em que o coeficiente angular é numericamente igual ao coeficiente de expansão térmica $(\beta)$.

Santos e Vieira (2010) comentam que a partir do conhecimento do coeficiente de expansão térmica é possível estimar outras propriedades físico-químicas (entre elas, a entalpia de vaporização) e avaliar os impactos no sistema de medição volumétrico.

Bejan (1996) comenta sobre a influência do coeficiente de expansão térmica no estudo da convecção natural de um fluido em escoamento através do grupo adimensional de Rayleigh ( $R a)$, conforme indicado na equação (3).

$$
R a=\frac{g \cdot \beta \cdot\left(T_{w}-T \infty\right) \cdot y}{\alpha \cdot \eta}
$$

Em que $g$ corresponde à aceleração da gravidade; $T_{w}$ e $T_{\infty}$ correspondem, respectivamente, às temperaturas da superfície e do fluido distante da superfície; $y$, a altura; e, $\alpha$ e $\eta$ correspondem, respectivamente, à difusividade térmica e à viscosidade cinemática.

Young e Freedman (2008) comentam da utilização do coeficiente de expansão térmica no cálculo da tensão volumétrica provocada por um aumento de temperatura $(\Delta p)$. Considere que a temperatura de um material sob pressão aumente, entretanto se este material for impedido de se dilatar, irá existir um aumento de pressão. Este aumento de pressão é chamado de tensão 
volumétrica provocada por um aumento de temperatura. A equação (4) indica o cálculo dessa grandeza.

$$
\Delta p=B \cdot \beta \cdot \Delta T
$$

Em que $B$ e $\Delta T$ correspondem, respectivamente, ao módulo de compressão e à variação de temperatura.

O objetivo deste trabalho foi verificar a existência de uma correlação linear entre o coeficiente de expansão térmica do suco de abacaxi $(\beta)$ e o teor de sólidos totais $\left(X_{s}\right)$. Para tanto, o coeficiente de expansão térmica foi obtido a partir da modelagem matemática de dados da densidade do suco de abacaxi em função da temperatura e do teor de sólidos totais. A modelagem matemática foi desenvolvida por Cabral e colaboradores (2010) utilizando suco de abacaxi produzido a partir de amostras da variedade Smooth Cayenne, comercializadas no município de São José do Rio Preto (SP).

\section{Material e Métodos}

Cabral e colaboradores (2010) realizaram uma modelagem matemática de dados experimentais da densidade do suco de abacaxi $(\rho)$ em função da temperatura $(T)$ e do teor de sólidos totais $\left(X_{s}\right)$. A equação encontrada corresponde à equação (5).

$$
\rho=998-0,35 \cdot T+4,71 \cdot X_{s}
$$

A equação (5) considerou o teor de sólidos totais variando de 11 a 52,4º Brix e temperaturas no intervalo de 17,4 a $85,8^{\circ} \mathrm{C}$.

Nesta equação, as unidades da densidade, da temperatura e do teor de sólidos totais são, respectivamente, $\mathrm{kg} . \mathrm{m}^{-3},{ }^{\circ} \mathrm{C} \mathrm{e}^{\circ} \mathrm{Brix}$.

Os valores do teor de sólidos totais $\left(X_{s}\right)$ foram $11^{\circ} \mathrm{Brix}$; $17,7^{\circ} \mathrm{Brix} ; 23,5^{\circ} \mathrm{Brix}$; $33,2^{\circ} \mathrm{Brix}$; $37,9^{\circ}$ Brix; $43,2^{\circ}$ Brix e $52,4^{\circ}$ Brix. Enquanto que da temperatura $(T)$ foram $17,4{ }^{\circ} \mathrm{C} ; 36,7^{\circ} \mathrm{C} ; 54,8$ ${ }^{\circ} \mathrm{C} ; 76,2{ }^{\circ} \mathrm{C}$ e $85,8{ }^{\circ} \mathrm{C}$. Todos estes valores estão indicados no trabalho de Cabral e colaboradores (2010).

A Tabela 1 indica os valores calculados para a densidade do suco de abacaxi $(\rho)$ em função do teor de sólidos totais $\left(X_{s}\right)$ e da temperatura $(T)$.

Com base nos valores da densidade $(\rho)$ e da temperatura $(T)$ indicados na Tabela 1 , foi possível realizar uma análise de regressão para o cálculo do coeficiente de expansão térmica ( $\beta$ ). 
Nesta análise foram utilizadas as equações de (6) a (13) que determinam o coeficiente de expansão térmica $(\beta)$ e o coeficiente de correlação $\left(r^{2}\right)$ para os dados emparelhados de $\ln \left(\rho_{0} / \rho\right)$ e $\left(T-T_{0}\right)$.

Tabela 1 - Valores da densidade do suco de abacaxi em função da temperatura e do teor de sólidos totais

\begin{tabular}{cccccccc}
\hline$X_{s}$ & $11^{\circ}$ Brix & $17,7^{\circ}$ Brix & $23,5^{\circ}$ Brix & $33,2^{\circ}$ Brix & $37,9^{\circ}$ Brix & $43,2^{\circ}$ Brix & $52,4^{\circ}$ Brix \\
\hline$T\left({ }^{\circ} \mathrm{C}\right)$ & $\rho\left(\mathrm{kg} \cdot \mathrm{m}^{-3}\right)$ & $\rho\left(\mathrm{kg} \cdot \mathrm{m}^{-3}\right)$ & $\rho\left(\mathrm{kg} \cdot \mathrm{m}^{-3}\right)$ & $\rho\left(\mathrm{kg} \cdot \mathrm{m}^{-3}\right)$ & $\rho\left(\mathrm{kg} \cdot \mathrm{m}^{-3}\right)$ & $\rho\left(\mathrm{kg} \cdot \mathrm{m}^{-3}\right)$ & $\rho\left(\mathrm{kg} \cdot \mathrm{m}^{-3}\right)$ \\
\hline 17,4 & 1043,720 & 1075,277 & 1102,595 & 1148,282 & 1170,419 & 1195,382 & 1238,714 \\
36,7 & 1036,965 & 1068,522 & 1095,840 & 1141,527 & 1163,664 & 1188,627 & 1231,959 \\
54,8 & 1030,630 & 1062,187 & 1089,505 & 1135,192 & 1157,329 & 1182,292 & 1225,624 \\
76,2 & 1023,140 & 1054,697 & 1082,015 & 1127,702 & 1149,839 & 1174,802 & 1218,134 \\
85,8 & 1019,780 & 1051,337 & 1078,655 & 1124,342 & 1146,479 & 1171,442 & 1214,774 \\
\hline
\end{tabular}

$$
\beta=\left\{\frac{n \cdot a-b}{n \cdot c-d}\right\}
$$

Em que:

$$
\begin{aligned}
a & =\left\{\sum\left[\left(T-T_{0}\right) \cdot \ln \left(\rho_{0} / \rho\right)\right]\right\} \\
b & =\left\{\sum\left(T-T_{0}\right)\right\} \cdot\left\{\sum \ln \left(\rho_{0} / \rho\right)\right\} \\
c & =\left\{\sum\left(T-T_{0}\right)^{2}\right\} \\
d & =\left\{\sum\left(T-T_{0}\right)\right\}^{2}
\end{aligned}
$$

Sendo $n$ correspondente ao número de dados amostrais emparelhados de $\ln \left(\rho_{0} / \rho\right)$ e $\left(T-T_{0}\right)$.

$$
r^{2}=\frac{(n \cdot a-b)}{\{\sqrt{(n \cdot c-d)}\} \cdot\{\sqrt{(n \cdot e-f)}\}}
$$

Em que:

$$
\begin{aligned}
& e=\sum\left\{\ln \left(\rho_{0} / \rho\right)\right\}^{2} \\
& f=\left\{\sum \ln \left(\rho_{0} / \rho\right)\right\}^{2}
\end{aligned}
$$

Com base na Tabela 1, para cada teor de sólidos totais, $n$ é igual a 5 .

Nos cálculos, o valor considerado para $T_{0}$ corresponde a $17,4^{\circ} \mathrm{C}$ e a densidade inicial $\left(\rho_{0}\right)$, ao valor da densidade nesta temperatura

Para verificar se existe uma correlação linear entre os coeficientes de expansão térmica $(\beta)$ e o teor de sólidos totais ( $X_{s}$ ) foi realizada uma análise de regressão linear dos dados emparelhados de $\beta$ e $X_{s}$. 
A equação de correlação linear entre os coeficientes de expansão térmica e o teor de sólidos totais é dada pela equação (14), em que $a_{0}$ corresponde ao coeficiente angular, enquanto que $b_{0}$ corresponde ao coeficiente linear.

$$
\beta=a_{0} \cdot X_{s}+b_{0}
$$

Em que:

$$
\begin{aligned}
& a_{0}=\left\{\frac{n_{0} \cdot h-i}{n_{0} \cdot j-k}\right\} \\
& b_{0}=\left\{\frac{p-q}{n_{0} \cdot j-k}\right\} \\
& h=\sum\left(X_{s} \cdot \beta\right) \\
& i=\left(\sum X_{s}\right) \cdot\left(\sum \beta\right) \\
& j=\sum X_{s}^{2} \\
& k=\left(\sum X_{s}\right)^{2} \\
& p=\left(\sum \beta\right) \cdot \sum X_{s}^{2} \\
& q=\left\{\sum\left(X_{s} \cdot \beta\right)\right\} \cdot \sum X_{s}
\end{aligned}
$$

Sendo $n_{0}$ correspondente ao número de dados amostrais emparelhados de $\beta$ e $X_{s}$. Neste caso, $n_{0}$ é igual a 7 .

O coeficiente de correlação $\left(R^{2}\right)$ para os dados emparelhados de $\beta$ e $X_{s}$ é dado pelas equações (23), (24) e (25).

$$
R^{2}=\frac{n_{0} \cdot h-i}{\left\{\sqrt{\left(n_{0} \cdot j-k\right)}\right\} \cdot\left\{\sqrt{\left(n_{0} \cdot l-m\right)}\right\}}
$$

Em que:

$$
\begin{aligned}
& l=\sum \beta^{2} \\
& m=\left(\sum \beta\right)^{2}
\end{aligned}
$$

As equações de (6) a (25) foram adaptadas de Triola (2008).

\section{Resultados e Discussão}


A Tabela 2 relaciona os valores do coeficiente de expansão térmica ( $\beta$ ) para o suco de abacaxi e os coeficientes de correlação $\left(r^{2}\right)$ obtidos através da análise de regressão linear dos dados emparelhados de $\ln \left(\rho_{o} / \rho\right)$ e $\left(T-T_{0}\right)$.

Tabela 2 - Resultados da análise de regressão linear dos dados emparelhados de $\ln \left(\rho_{o} / \rho\right)$ e $\left(T-T_{0}\right)$.

\begin{tabular}{ccc}
\hline$X_{s}$ & $\beta\left({ }^{\mathbf{o}} \mathbf{C}^{-\mathbf{1}}\right) \mathbf{\times} \mathbf{1 0}^{-4}$ & $r^{2}$ \\
\hline 11 & 3,3930 & 0,9999 \\
17,7 & 3,2923 & 0,9999 \\
23,5 & 3,2098 & 0,9999 \\
33,2 & 3,0808 & 0,9999 \\
37,9 & 3,0219 & 0,9999 \\
43,2 & 2,9581 & 0,9999 \\
52,4 & 2,8536 & 0,9999 \\
\hline
\end{tabular}

O gráfico de $\ln \left(\rho_{0} / \rho\right)$ versus $\left(T-T_{0}\right)$ para os dados indicados na Tabela 1 está representado na Figura 1.

Figura 1 - Gráfico de $\ln \left(\rho_{0} / \rho\right)$ versus $\left(T-T_{0}\right)$ para o suco de abacaxi

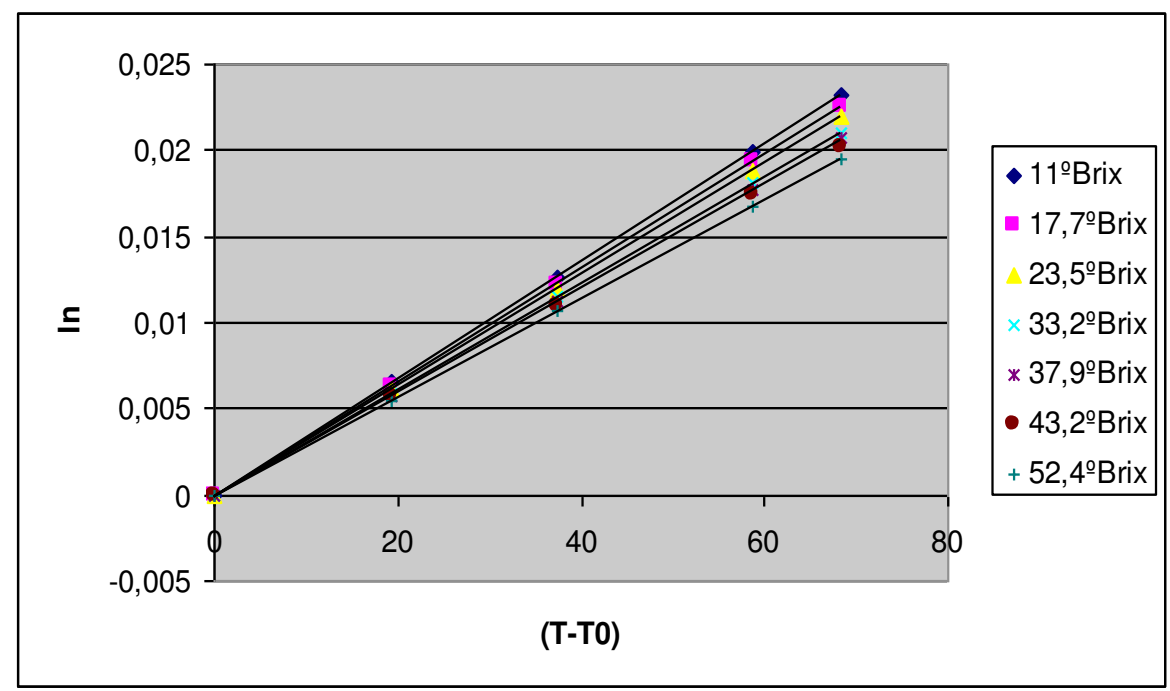

Santos e Vieira (2010) comentam que os valores do coeficiente de expansão estão relacionados com a energia de ligação química entre as partículas, de maneira que materiais em que as ligações químicas são fortes apresentam baixos coeficientes de expansão térmica.

A dilatação térmica está associada à variação assimétrica da energia de ligação com a distância entre as partículas. Ou seja, durante o aquecimento estas partículas aumentam a frequência e a amplitude de vibração e como as forças de repulsão são sempre maiores que as de atração, a distância média entre elas também aumenta (PADILHA, 1997).

De acordo com Cabral e Lago (2002), o coeficiente de expansão térmica da água equivale a $2,07 \times 10^{-4 o} \mathrm{C}^{-1}$. 
A molécula de água é uma molécula polar, onde a interação entre suas moléculas é do tipo ligação de hidrogênio. A ligação de hidrogênio ocorre quando um átomo de hidrogênio liga-se por covalência a um átomo mais eletronegativo e mantém uma afinidade residual por outro átomo eletronegativo, apresentando uma tendência à carga positiva (BROWN; HOLME, 2009).

Moléculas de glicose, frutose e sacarose apresentam em sua estrutura grupamentos hidroxila, que são polares, pois o oxigênio é mais eletronegativo do que o hidrogênio. No estado sólido, as moléculas desses açúcares encontram-se ligadas umas às outras através das ligações de hidrogênio. Quando ocorre a dissolução desses açúcares na água, são estabelecidas novas ligações de hidrogênio entre os grupamentos hidroxila das moléculas de sacarose e as moléculas de água, o que garante a dissolução destes açúcares na água. Entretanto, também se formam forças intermoleculares do tipo dipolo permanente-dipolo permanente entre as moléculas destes açúcares e da água (PERUZZO; CANTO, 2010).

As ligações de hidrogênio, quando comparadas com as forças intermoleculares do tipo dipolo permanente-dipolo permanente, são mais fortes (BROWN; HOLME, 2009).

Talvez por este motivo, o coeficiente de expansão térmica da água seja menor que, por exemplo, o coeficiente de expansão térmica do suco de abacaxi com $11^{\circ}$ Brix.

$\mathrm{Na}$ água ocorrem exclusivamente ligações de hidrogênio, quando comparadas com o suco de abacaxi com $11^{\circ}$ Brix.

No suco de abacaxi, os compostos dissolvidos interagem com as moléculas de água por ligações de hidrogênio e forças intermoleculares do tipo dipolo permanente-dipolo permanente.

Questionando o fato de que à medida que se aumenta o teor de sólidos totais, o coeficiente de expansão térmica diminui. Talvez isto esteja associado à interação que ocorre entre os compostos dissolvidos no suco de abacaxi.

Como a maioria do teor de sólidos totais no suco de abacaxi corresponde a açúcares (glicose, frutose e sacarose), à medida que o teor de sólidos totais aumenta, mais a solução fica concentrada e mais moléculas de açúcares interagem entre si. Assim, em sucos com maior teor de sólidos totais, ocorrem com maior frequência ligações de hidrogênio entre as moléculas de açúcares. A formação de ligações de hidrogênio entre as moléculas de açúcares (interações mais fortes) faz com que o coeficiente de expansão térmica diminua.

Todas estas considerações necessitam ser mais estudadas e discutidas.

Segundo Santos e Vieira (2010), a partir do conhecimento do coeficiente de expansão térmica é possível avaliar os impactos no sistema de medição volumétrico.

Dessa forma, considerando um volume de 1000 litros de água com uma variação de temperatura de $10^{\circ} \mathrm{C}$, o volume de água aumenta em torno de 2,1 litros. Com este mesmo raciocínio, 
o volume de 1000 litros de suco de abacaxi com $11^{\circ}$ Brix, com uma variação de temperatura de $10^{\circ} \mathrm{C}$, o volume desse suco aumenta em torno de 3,4 litros.

A Tabela 3 relaciona a ordem de aumento de volume para uma variação de temperatura de $10^{\circ} \mathrm{C}$, considerando um volume de 1000 litros para cada um dos sucos de abacaxi estudados.

Tabela 3 - Ordem de aumento de volume em função do teor de sólidos totais

\begin{tabular}{cc}
\hline$X_{s}$ & Ordem de aumento (L) \\
\hline 11 & 3,4 \\
17,7 & 3,3 \\
23,5 & 3,2 \\
33,2 & 3,1 \\
37,9 & 3,0 \\
43,2 & 3,0 \\
52,4 & 2,9 \\
\hline
\end{tabular}

Não foi encontrado na literatura, nenhum trabalho estudando o coeficiente de expansão térmica de sucos tropicais.

Zuritz e colaboradores (2005) estudaram o coeficiente de expansão térmica de sucos clarificados de uva (fruta considerada de clima temperado) e determinaram uma expressão do coeficiente de expansão térmica em função do teor de sólidos totais e da temperatura. Esta expressão é uma expressão polinomial de grau 2, tanto para a temperatura como para o teor de sólidos totais.

A partir das equações (15) a (25), foi possível verificar a existência de uma correlação linear entre o coeficiente de expansão térmica $(\beta)$ e o teor de sólidos totais $\left(X_{s}\right)$.

$\mathrm{Na}$ análise de regressão linear dos dados do coeficiente de expansão térmica $(\beta)$ e o teor de sólidos totais $\left(X_{s}\right)$, o valor encontrado para o coeficiente de correlação $\left(R^{2}\right)$ foi de $-0,9987$, em que $a_{o}$ e $b_{0}$ correspondem, respectivamente, a $3,5237 \times 10^{-4} \mathrm{e}-1,3048 \times 10^{-6}$.

Assim, a equação (14) fica reescrita na forma de:

$$
\beta=3,5237 \times 10^{-4}-1,3048 \times 10^{-6} \cdot X_{s}
$$

A equação (26) é válida para temperaturas que vão de 17,4 a $85,8^{\circ} \mathrm{C}$, em que o teor de sólidos totais $\left(X_{s}\right)$ varia de 11 a $52,4^{\circ}$ Brix.

Triola (2008) associa que correlações lineares negativas, o valor do coeficiente de correlação também é negativo, e vice-versa.

O gráfico de $\beta$ versus $X_{s}$, ilustrado na Figura 3, demonstra a situação de correlação linear negativa. 


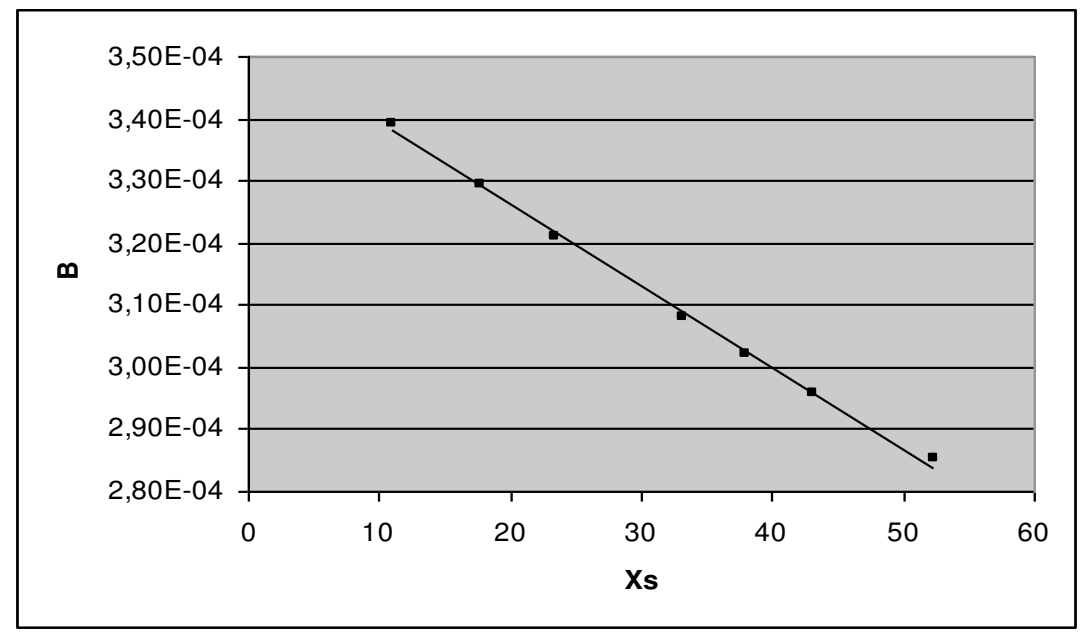

Toledo e Ovalle (1985) comentam que o módulo do coeficiente de correlação mede o grau de relacionamento linear entre os valores emparelhados das variáveis dependente e independente em uma amostra, avaliando assim, a "qualidade" do ajuste. Ou seja, quanto mais próximo for da unidade, melhor é a "qualidade" do ajuste da função aos pontos do diagrama de dispersão.

Lira (2004) expressa que para valores do módulo do coeficiente de correlação maiores ou iguais a 0,99 e menores que 1,0, a correlação linear é classificada como muito forte.

Dessa forma, a correlação linear entre o coeficiente de expansão térmica e o teor de sólidos totais é classificado como muito forte.

\section{Conclusão}

Os resultados sugerem que os valores do coeficiente de expansão térmica do suco de abacaxi são maiores que o coeficiente de expansão térmica da água e que à medida que se aumenta o teor de sólidos totais, o coeficiente de expansão térmica diminui.

A análise de regressão linear entre dados do coeficiente de expansão térmica e o teor de sólidos forneceu um coeficiente de correlação, em módulo, bem próximo a unidade, indicando "qualidade" no ajuste da função aos pontos de dispersão e uma correlação linear negativa classificada como muito forte.

\footnotetext{
Abstract

The knowledge of the thermal expansion coefficient of a substance is important to evaluate the impact of thermal expansion of the volumetric measurement. For products of tropical fruits, such as pineapple juice, there are not references in the literature on the thermal expansion coefficient. In this sense, this study aimed to determine whether a linear correlation exists between the thermal expansion coefficient of pineapple juice and the total solids content. Thus, the thermal expansion coefficient was obtained from the mathematical modeling of the data density of pineapple juice as a function of temperature and total solids content. The thermal expansion coefficient ranged from $2.8536 \times 10^{-4}$ to $3.3930 \times 10^{-4 o} \mathrm{C}^{-1}$ in a range of total solids content from 11 to $52,4^{\circ}$ Brix. In the same range of total solids content, it was found that there is a linear relationship between the thermal expansion coefficient and total solids content, with a correlation coeficient close to unity, classifying as very strong linear correlation.
} 
Key-words: dilatation, total solids content, correlation.

\section{Referências}

BEJAN, A. Transferência de calor. São Paulo: Edgard Blücher, 1996.

BROWN, L. S.; HOLME, T. A. Química geral aplicada à Engenharia. São Paulo: Cengage Learning, 2009.

CABRAL, R.A.F.; GUT, J.A.W.; TELIS, V.R.N.; TELIS-ROMERO, J. Non-newtonian flow and pressure drop of pineapple juice in a plate heat exchanger. Brazilian Journal of Chemical Engineering, v. 27, n. 4, p. 563-571, 2010. http://dx.doi.org/10.1590/S0104-66322010000400008

CABRAL, F.; LAGO, A. Física 2. São Pualo: Harbra Ltda., 2002.

CALLISTER, W. D. Ciência e Engenharia de Materiais: uma introdução. Rio de Janeiro: LTC Ltda., 2008.

CANCIAM, C. A. Predição do coeficiente de expansão térmica do óleo de algodão. Revista Publicatio UEPGCiências Exatas e da Terra, Ciências Agrárias e Engenharias, v. 11, n 3, p. 27-31, 2005.

CANCIAM, C.A. Predição do coeficiente de expansão térmica do óleo de gergelim (Sesamum indicum L.) através da aplicação de regressão linear. Revista e-xacta, v. 3, n 1, 2010.

CHAVES, M.C.V.; GOUVEIA, J.P.G.; ALMEIDA, F.A.C.; LEITE, J.C.A.; SILVA, F.L.H. Características físicoquímicas do suco de acerola. Revista de Biologia e Ciências da Terra, v. 4, n. 2, 2004

COUTO, D.S. Avaliação da qualidade de suco de abacaxi (Ananas comosus L. Merr cv. Smooth Cayenne) concnetrado por osmose inversa. Seropédica, 2008. 60 f. Dissertação (Mestrado em Ciência e Tecnologia de Alimentos), Universidade Federal Rural do Rio de Janeiro.

GRANADA, G.G.; ZAMBIAZI, R.C.; MENDONÇA, C.R.B. Abacaxi: produção, mercado e subprodutos. Boletim do CEPPA, v. 22, n. 2, p. 405-422, 2003.

INCROPERA, F. P.; DEWITT, D. P. Fundamentos de Transferência de Calor e de Massa. Rio de Janeiro: LTC Ltda., 2008.

LIRA, S. A. Análise de correlação: abordagem teórica e de construção dos coeficientes com aplicação. Curitiba, 2004. 196 f. Dissertação (Mestrado em Ciências) - Curso de Pós-Graduação em Métodos Numéricos em Engenharia Universidade Federal do Paraná.

MARCELLINI, P.S.; DELIZA, R.; BOLINI, H.M.A. Caracterização sensorial de suco de abacaxi concentrado, reconstituído e adoçado com diferentes edulcorantes e sacarose. Alimentos e Nutrição, v. 17, n. 2, p. 143-50, 2006.

MARIN, J.O.B.; CARVALHO, S.O.; PRADO, L.A.; PEREIRA, J.M. Panorama geral da produção de abacaxi e comportamento sazonal de preços do abacaxi "Pérola" comercializado em Goiás, disponível em: $<\mathrm{http}$ ///www.sober.org.br/palestra/9/550.pdf> . Acesso em 05 mai. 2012.

PADILHA, A. F. Materiais de Engenharia: microestrutura e propriedades. São Paulo: Hemus Ltda., 1997.

PERUZZO, F.M.; CANTO, E.L. Química na abordagem do cotidiano - volume 1: Química Geral e Inorgânica. São Paulo: Moderna, 2010.

PINHEIRO, A.M.; FERNANDES, A.G.; FAI, A.E.C.; PRADO, G.M.; SOUSA, P.H.M.; MAIA, G.A. Avaliação química, físico-química e microbiológica de sucos de frutas integrais: abacaxi, caju e maracujá. Ciência e Tecnologia de Alimentos, v. 26, n. 1, p. 98-103, 2006. http://dx.doi.org/10.1590/S0101-20612006000100017

REINHARDT, D.H.; MEDINA, V.M.; CALDAS, R.C.; CUNHA, G.A.P.; ESTEVAM, R.F.H. Gradientes de qualidade em abacaxi "Pérola" em função do tamanho e do estágio de maturação do fruto. Revista Brasileira de Fruticultura, v. 26, n. 3, p. 544-546, 2004. http://dx.doi.org/10.1590/S0100-29452004000300041 
SANTOS, D. Q.; VIEIRA, D. F. Determinação de coeficiente de expansão térmica do biodiesel e seus impactos no sistema de medição volumétrico. Eclética Química, v. 35, n 04, p. 107-112, 2010. http://dx.doi.org/10.1590/S010046702010000400014

TOLEDO, G. L.; OVALLE, I. I. Estatística Básica. São Paulo: Atlas Ltda., 1985.

TRIOLA, M. F. Introdução à Estatística. Rio de Janeiro: Livros Técnicos e Científicos Ltda., 2008.

YOUNG, H.D.; FREEDMAN, R.A. Física III - Termodinâmica e Ondas. São Paulo: Addison Wesley, 2008.

ZURITZ, C.A.; PUNTES, E.M.; MATHEY, H.H.; PÉREZ, E.H.; GASCÓN, A.; RUBIO, L.A.; CARULlO, C.A.; CHERNIKOFF, R.E.; CABEZA, M.S. Density, viscosity and coefficient of thermal expansion of clear grape juice at different solubles solid concentrations and temperatures. Journal of Food Engineering, v. 71, p. 143-149, 2005. http://dx.doi.org/10.1016/j.jfoodeng.2004.10.026

Submetido em 10 jun. 2012, Aceito para publicação em 16 jul. 2013. 